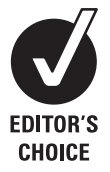

Correspondence to:

Professor R Jones, King's

College London, Department of

General Practice \& Primary Care,

5 Lambeth Walk, London SE11

6SP, UK; roger.jones@kcl.ac.uk

Received 8 February 2008

Accepted 29 June 2008

\title{
Primary care research and clinical practice: gastroenterology
}

\author{
$\mathrm{R}$ Jones
}

\begin{abstract}
Gastrointestinal problems account for a significant proportion of general practitioners' workload, and gastrointestinal cancers, taken together, make up the largest group of malignancies. Approximately $10 \%$ of consultations in general practice in the UK are for gastrointestinal symptoms or problems, split roughly equally between the upper and lower gastrointestinal

has generated a great deal of important information about the epidemiology, natural history, diagnosis and management of many of the common gastrointestinal disorders. This paper sets out to describe some of the research that has been undertaken in primary care, its contribution to clinical practice, and important areas for future research.
\end{abstract} tract. Gastroenterology represents about 10\% of the work of hospital specialists and the prescribing costs involved in the management of gastrointestinal disorders in general practice are around $14 \%$ of the drug budget. These disorders range from relatively minor and self limiting conditions such as acute gastritis and acute gastroenteritis, through the more significant, chronic digestive disorders such as gastro-oesophageal reflux disease (GORD), irritable bowel syndrome (IBS) and coeliac disease, to much more serious problems including inflammatory bowel disease (IBD) and upper gastrointestinal and colorectal cancer.

Gastrointestinal problems account for a significant proportion of the workload of general practitioners (GPs), and gastrointestinal cancers, taken together, make up the largest group of malignancies. GPs have important roles in early diagnostic decision making, separating the minority of patients with potential alarm symptoms, suggestive of serious disease and mandating early investigation, from the majority whose symptoms are less significant and whose conditions may well resolve spontaneously or with minimal intervention. GPs and their teams have the responsibility for the long term care of many of the chronic gastrointestinal disorders, including gastro-oesophageal reflux disease (GORD), irritable bowel syndrome (IBS), coeliac disease and inflammatory bowel disease (IBD), and general practice has a potentially important role in screening for colorectal cancer, although this has not yet been fully realised. However, there is evidence from surveys undertaken in the $\mathrm{UK}^{1}$ and Europe ${ }^{2}$ that the management of the common gastrointestinal disorders by GPs is inconsistent and often does not conform to existing evidence or evidence based guidelines. Organisations such as the British Society of Gastroenterology (BSG) and the Primary Care Society for Gastroenterology (PCSG) produce such guidance, which is also available through Cochrane reviews and sources such as Clinical Evidence.

Until perhaps 20 years ago, most primary care management of digestive disorders relied heavily on evidence collected in hospital based studies, or on no evidence at all. Over the last two decades, however, primary care research in gastroenterology

\section{THE GENERAL PRACTICE CONTEXT}

The results of clinical research undertaken in general practice need to be interpreted against a background of a changing National Health Service (NHS), with new and emerging roles for GPs, as chronic disease management and other aspects of patient care continue to shift from the secondary to the primary care sector. For many years GPs have been involved in hospital endoscopy, and there are now at least $400 \mathrm{GP}$ endoscopists working around the country. The General Practitioners with Special Interests Scheme, established by the Department of Health and the Royal College of General Practitioners, ${ }^{3}$ provides a framework for the development of specialist interests in gastroenterology, and this trend is likely to continue.

The ways in which primary care services are delivered are beginning to change fundamentally. Private provision of primary care, in direct competition with traditional NHS providers, is now encouraged, and there is a strong policy move towards increasing centralisation of GP and other community services in larger groupings in newly equipped centres known as polyclinics. ${ }^{4}$

A further important consideration is the introduction of a pay-for-performance component into the contractual arrangements for GPs, through the introduction of the Quality and Outcomes Framework (QOF). ${ }^{5}$ The QOF provides financial incentives for the attainment of specified targets in the management of a number of chronic disorders, including diabetes, asthma, stroke and hypertension, but not, at present, for any gastrointestinal disorders. The QOF has, in a sense, partially solved the problem of implementation of clinical practice guidelines by providing direct financial rewards for their incorporation into routine general practice care.

\section{RESEARCH METHODS IN PRIMARY CARE GASTROENTEROLOGY}

Research publications over the last 20 years exemplify a range of methodologies used in the study of gastrointestinal problems in general practice. Epidemiological survey methods have been used effectively because of the accurate denominator provided by GPs' registered lists of patients, 
accompanied by the general public's willingness to respond to postal questionnaires measuring the prevalence and characteristics of gastrointestinal symptoms or disorders. Follow ups to these cross sectional surveys, less frequently performed, can provide clues to the natural history of common gastrointestinal symptoms. The behaviour of patients (such as factors affecting their likelihood to consult with gastrointestinal disorders) and physicians (such as their patterns and rates of usage of investigations and choice of drug treatments) have been studied by a combination of quantitative and qualitative (interview and narrative) methods, while clinical trials of therapeutic interventions have been conducted in a number of disease areas. The potential of large databases, such as the General Practice Research Database (GPRD) which is the world's largest primary care database, containing information on approximately 13 million patient years, has been recognised for some time, and a number of important studies have been published on the natural history of gastrointestinal disorders, patterns of treatment, outcomes of treatment, and the significance of alarm symptoms in early cancer diagnosis, as described later. Finally, GPs have frequently been involved in systematic reviews and meta-analyses of published studies which have attempted to identify aggregate findings of particular interest in the management of gastrointestinal disorders in general practice. ${ }^{67}$

\section{KEY RESEARCH FINDINGS \\ Epidemiology}

The earliest epidemiological studies in primary care gastroenterology were published in the mid 1980s and described the pattern and prevalence of dyspepsia, ${ }^{8}{ }^{9} \mathrm{IBS}^{10}$ and rectal bleeding ${ }^{11}$ in the general population, using general practice lists as the data source for the denominator and postal questionnaires sent to a stratified sample of subjects (patients) as the numerator. As well as providing important information about the ubiquity and the demographic and other factors associated with common gastrointestinal disorders, these studies began to raise the awareness of the importance of understanding patients' decisions to consult clinicians about common symptoms. In the case of dyspepsia, for example, while around $20 \%$ of the population experience dyspeptic symptoms, only about a quarter to one third of these patients ever seek medical advice for them. Similar findings emerged in studies on IBS and rectal bleeding and these results have influenced the way in which secondary care colleagues' view the consulting patient population. Epidemiological work in coeliac disease emphasised the extent to which this important condition is under-recognised and under-diagnosed in general practice, ${ }^{12}$ and these early papers have undoubtedly had a significant impact on raising awareness of the condition itself and of the symptomatic and clinical factors which place patients at increased risk. Epidemiological work on IBD showed the condition to be considerably more common than previously estimated from hospital based studies, with careful general practice based studies defining precise prevalence rates for ulcerative colitis and Crohn's disease ${ }^{13}$ and their impact on patients. ${ }^{14}$

\section{Natural history}

The great pioneer of natural history studies in general practice was John Fry, whose painstaking manual recording of his patients' consultations over a period of many years provided early and accurate data on the incidence and prevalence of many important conditions, particularly peptic ulcer disease, and of their progression over time. ${ }^{15}$ Population based studies of the natural history of common gastrointestinal disorders are less frequent, although useful work on dyspepsia and IBS has been undertaken in the UK, Sweden and the Netherlands using repeated cross-sectional population surveys. ${ }^{16-18}$ Findings from these studies include the observation that the majority of patients presenting in general practice with abdominal pain improve, with symptom resolution in $68 \%$ at 12 months, and that functional diagnoses such as IBS and non-ulcer dyspepsia frequently overlap and are unstable, with patients moving from one functional diagnostic group to another over a 12 month observation period. Recently the use of the GPRD has allowed, through long term (retrospective) data analysis, a clearer understanding and, in some ways, a more precise quantitative appreciation of the prevalence and progression of conditions such as dyspepsia and IBS. ${ }^{19}{ }^{20}$ The GPRD has also been used to examine the associations between various disorders, such as that between psychological factors and $\mathrm{IBS}^{20}$ and GORD and asthma. ${ }^{21}$ Anxiety and depression continue to be prevalent comorbidities in IBS. Before diagnosis prevalence of depression is $13 \%$, compared with $5 \%$ in controls, rising to $20 \%$ vs $10 \%$ after diagnosis. Corresponding figures for anxiety are $11 \%$ before diagnosis and $13 \%$ after diagnosis, compared to $6 \%$ and $7 \%$, respectively, in non-IBS controls. There are clear associations between asthma and GORD, so that patients with a diagnosis of asthma have an increased risk (odds ratio 1.5, $\mathrm{p}<0.01$ ) of developing reflux in a 3 year follow-up period, with a non-significant trend towards an increased rate of asthma diagnoses in patients with an established diagnosis of GORD.

\section{Patient and professional behaviour}

Some of the most influential research in primary care gastroenterology has been concerned with individuals' decisions to consult primary care physicians with digestive symptoms. Most digestive symptoms and disorders are common in the general population, with some $20 \%$ of subjects reporting dyspepsia, $15 \%$ IBS and 20\% GORD in any 12 month period, but only a minority of these patients seek medical advice. The finding that psychological and emotional concerns, including fears of cancer and heart disease and beliefs that their symptoms might represent serious disease, are more important than symptom severity in dyspeptic patients' decisions to consult were striking findings, ${ }^{22}$ which have been replicated in conditions such as IBS $^{23}$ and rectal bleeding. ${ }^{11}$ The influence of more formal psychological and psychiatric disorders-anxiety and depression-are somewhat controversial. Early reports suggested that patients with dyspepsia and IBS who reported more psychosocial morbidity were more likely to consult than those without, but more recent studies, particularly in Australia and North America, have found a lesser effect of psychological problems in the consultation decision. ${ }^{24} 25$

The behaviour of clinicians has also been a subject of research interest, particularly in relation to their variability in the use of Helicobacter pylori testing and eradication in dyspepsia, or in their rates of prescription of proton pump inhibitors. ${ }^{26}$ Qualitative methods have been used to improve our understanding of the factors affecting behaviour change among GPs and the ways in which new management or prescribing habits are sustained and embedded. ${ }^{27}{ }^{28}$ These studies have emphasised the role of therapeutic experiences with individual patients in determining prescribing behaviour and the relative importance of influences such as publications, continuing medical education and academic detailing on clinicians' uptake of new treatments. 


\section{Diagnosis}

Effective management of gastrointestinal disorders in general practice depends on GPs' appreciation of key diagnostic criteria, and there is growing evidence that accurate diagnosis and better clinical management can be achieved by the use of structured questionnaires and other instruments, such as the Reflux Disease Questionnaire (RDQ), ${ }^{29}$ the Gastro-oesophageal Reflux Disease Impact Scale (GIS), ${ }^{30}$ and the Rome Criteria for the diagnosis of IBS. ${ }^{31}$ While only a minority of GPs conduct upper and lower gastrointestinal endoscopy in their surgeries or other community settings, the majority of GPs in the UK now have direct access to upper endoscopy and, to a slightly lesser extent, to flexible sigmoidoscopy and colonoscopy in hospitals. H pylori serology and carbon urea breath testing are both readily available, although access to oesophageal manometry, $\mathrm{pH}$-metry and more sophisticated investigations requires consultant referral.

A good deal of research has been carried out on the ways in which GPs use various diagnostic approaches in gastroenterology, particularly endoscopy, but also $H$ pylori testing and empirical antisecretory therapy. Early studies of open access endoscopy for GPs emphasised that GPs used the services as "effectively" and responsibly as their hospital colleagues, as well as providing new information on the pattern and prevalence of endoscopically diagnosed gastrointestinal disorders in general practice populations. ${ }^{32}$ The recognition of the value of open access endoscopy in the mid 1980s led to a national move towards the provision of widespread open access for all GPs. In the pre- $H$ pylori era there was lively debate about the benefits and disbenefits of early endoscopy versus empirical antisecretory therapy, and valuable work in Denmark initially suggested that a prompt endoscopy strategy was likely to be more cost effective than the use of empirical $\mathrm{H}_{2}$ blockade. ${ }^{33}$ More recently the debate has centred around the relative values of $H$ pylori testing and endoscopy in the investigation and management of dyspepsia in general practice, and further trials from the Copenhagen group have confirmed the appropriateness of a "test and treat" strategy compared with early endoscopy or empirical therapy. ${ }^{34}$ However, as $H$ pylori infection becomes less of a problem in western European countries and non-helicobacter ulcers (that is, those caused by non-steroidal antiinflammatory drugs) become more important, these questions have somewhat less currency.

Work on the GPRD has also been valuable in relation to early diagnosis in primary care. A large study of several thousand patients presenting in general practice with alarm symptoms, including dysphagia and rectal bleeding, provided, for the first time, risk information about cancer diagnosis (and timing) in men and women in different age strata. ${ }^{35}$ Although the overall positive predictive values (PPVs) for a cancer diagnosis following presentation with alarm symptoms are relatively low (for example, haematuria has a PPV of only $3.4 \%$ for urinary tract cancer, and dysphagia a PPV of only $2.4 \%$ for oesophageal cancer in women) in particular groups, notably elderly men, predictive values for a cancer diagnosis within the first 90 days of presentation rise steeply (for example, haemoptysis has a PPV of $17 \%$ for lung cancer in men over 75 years of age, and dysphagia a PPV of 9\% for oesophageal cancer in men over 65). These results indicate that urgent investigation in at-risk groups is justified, while sounding a word of caution about overinvestigation in lower risk patients.

\section{Treatment}

Most of the major trials of the drug treatment of peptic ulceration, GORD and IBD have been conducted in multicentre settings coordinated by hospital specialists, although a number of innovative studies of drug treatment have been undertaken in primary care. In the 1980s the Trondheim group pioneered the use of "n of 1" trials to examine the effect of antisecretory therapy in dyspepsia and to predict patients for whom antisecretory therapy is likely to be important. ${ }^{36}$ A number of non-endoscopic randomised controlled trials, using proton pump inhibitors, have emphasised the value of these agents in the empirical management of patients with upper gastrointestinal symptoms, ${ }^{37} 38$ while other work in primary care has documented the pattern of usage of PPIs and some of the factors associated with GPs' decisions to prescribe PPIs empirically or following upper gastrointestinal investigations. ${ }^{39}$

Although a number of randomised controlled trials (RCTs) of new agents developed for the treatment of IBS have been conducted in general practice, ${ }^{40}$ problems with side effects and efficacy have meant that most of these drugs are not available in the UK, and has led to a reorientation of the treatment of IBS towards non-drug treatments. Following on from encouraging work in hospital settings, a large RCT of cognitive behavioural therapy (CBT) for IBS in general practice showed a significant benefit of CBT when it was added to standard treatment with mebeverine, with beneficial symptomatic effects and a positive impact on work and social functioning persisting for between 3-6 months after a nurse delivered course of six CBT sessions. ${ }^{41}$ Cost effective analysis confirmed a modest increase in costs in the CBT group, ${ }^{42}$ and the recently published BSG guidelines on IBS include a recommendation that patients who respond unsatisfactorily to simple, initial treatment should be considered for CBT as part of their management. ${ }^{43}$

\section{RESEARCH AGENDA}

Early and accurate diagnosis of gastrointestinal disorders - the cancers and the non-malignant conditions-remains the most important goal. Delays in diagnosis of IBS, for example, are often associated with unnecessary referral to inappropriate specialists, leading to inappropriate surgical procedures such as hysterectomy ${ }^{44}$ and cholecystectomy. ${ }^{45}$ Delayed diagnosis or failure of diagnosis of coeliac disease and IBD are commonly reported and are likely to have significant adverse effects for patients, while the consequences of failing to detect cancer in patients presenting with alarm symptoms are self evident. The research agenda in these circumstances probably has less to do with an evidence gap than an implementation gap-most of the symptoms and risk factors associated with these conditions are fairly well documented, but it seems that accurate diagnosis is

\section{Current important research questions}

- How can GPs improve the timeliness and accuracy of diagnosis of gastrointestinal (Gl) cancer and other serious Gl disorders (such as coeliac disease and inflammatory bowel disease)?

- How can information technology best be used in the consultation to support high quality management of $\mathrm{Gl}$ disorders?

- How can the views and experiences of patients and service users best be incorporated into the development of meaningful outcomes for therapeutic trials and health services research?

- How can primary care best contribute to screening for colorectal cancer and to the care of survivors of colorectal cancer and other GI malignancies? 
difficult for many GPs, and it is worth thinking about the reasons for this. One is simply that gastrointestinal problems, although they account for 1 in 10 patients seen in our surgeries, do not carry the same "weight" or "threat" as the more traditional "killer" conditions such as stroke, coronary heart disease, diabetes and respiratory disease. As described above, the QOF offers a potentially powerful mechanism for cutting the "implementation corner", by providing natural incentives for the achievement of targets specified in financial practice guidelines, but does not yet include targets for gastrointestinal disorders. A national project currently underway, funded by the Health Foundation (http://www.kcl.ac.uk/depsta/medicine/ gppc/image/) is now attempting to develop quality criteria for the management of GORD, IBS, coeliac disease and IBD, based on a synthesis of patients' views and current best evidence and guidelines, and has the potential to inform future iterations of the QOF.

General practice in the UK does, however, have two very important advantages which should, potentially, support improved management of GI disorders, and these are the registered patient list and the sophistication of GP computer systems. Screening, surveillance, case finding, audit and review can all readily be undertaken using computerised records and databases, and most computer systems can readily be adapted to include "pop up" or "drop down" prompts and templates to encourage appropriate actions and appropriate surveillance of patients with acute and chronic presentations of GI problems. Modern primary care computer systems are also capable of producing high quality, personalised information for patients about gastrointestinal disorders. CORE, the digestive diseases charity, already produces an excellent range of well produced patient information leaflets which are available in paper and electronic format, and these have proven to be of great interest to patients taking part in recent focus group research on GI topics, at which they have also expressed a desire to have clearer explanation and ideas about prognosis, particularly in the chronic, functional GI disorders.

In terms of primary research, evaluation of the role of nondrug interventions in conditions including IBS, GORD, nonulcer dyspepsia and other functional bowel disorders is at an early stage in general practice; more trials are needed to define the place of the "talk therapies", including CBT, hypnotherapy and psychotherapy, either alone or as adjuncts to drug therapies, in the management of these conditions, which can sometimes prove refractory. The opportunity to deliver personalised gut orientated CBT via the internet is an exciting

\section{Key learning points}

- General practice requires a strong evidence base of research conducted in primary care to delivery high quality care for gastrointestinal (GI) disorders.

- Over the last 20 years numerous research studies in primary care have contributed to the evidence base. These include epidemiological (cross sectional and longitudinal) studies, clinical trials, evaluations of complex interventions, secondary analyses of large databases, and qualitative research.

- Understanding of natural history and management of many common Gl disorders such as dyspepsia, gastro-oesophageal reflux disease, irritable bowel syndrome, Helicobacter pylori infection, inflammatory bowel disease, and GI cancers has increased considerably as a result of this research.

\section{Contact information}

British Society of Gastroenterology: http://www.bsg.org.uk/

- Primary Care Society for Gastroenterology: http://www.pcsg. org.uk/

- European Society for Primary Care Gastroenterology: http:// www.espcg.org

- CORE, the Digestive Diseases Charity: http://www. corecharity.org.uk/

innovation worthy of careful study. The emerging structures within the UK Clinical Research Networks, including the local Primary Care Research Networks and the supporting Comprehensive Research Networks, provide a new impetus and infrastructure for the conduct of trials in general practice, and it will be important to make full use of these new opportunities.

Competing interests: The author is founding president of the PCSG and was founding chair of ESPCG.

\section{REFERENCES}

1. Jones R, Stevens R. Management of common gastrointestinal disorders in general practice: PCSG National Survey 2004. www.pcsg.org.uk.

2. Seifert B, Rubin G, de Wit N, et al. The management of common gastrointestinal disorders in general practice: a survey by the European Society for Primary Care Gastroenterology (ESPCG) in six European countries. Dig Liv Dis 2008;40:659-66.

3. Rosen R, Stevens R, Jones R. General practitioners with special clinical interests. BMJ 2003;327:460-2.

4. Finch R. When is a polyclinic not a polyclinic? BMJ 2008;336:916-8.

5. Majeed A, Lester $H$, Bindman A. Improving the quality of care with performance indicators. BMJ 2007;335:916-8.

6. Moayyedi P, Soo S, Deeks J, et al. Systematic review and economic evaluation of Helicobacter pylori eradication treatment for non-ulcer dyspepsia. Dyspepsia Review Group. BMJ 2000;321:659-64

7. Moayyedi P, Soo S, Deeks J, et al. Pharmacological interventions for non-ulcer dyspepsia. Cochrane Database of Systematic Reviews (4): CD001960, 2006.

8. Jones RH, Lydeard S. Prevalence of symptoms of dyspepsia in the community. BMJ 1989;298:30-2.

9. Jones RH, Lydeard S, Hobbs FDR, et al. Dyspepsia in England and Scotland. Gut 1990;31:401-5

10. Jones RH, Lydeard S. Irritable bowel syndrome in the general population. BMJ 1992;304:87-90.

11. Crosland $\mathbf{A}$, Jones R. Rectal bleeding: prevalence and consultation behaviour. BMJ 1995;311:486-8.

12. Hin $\mathbf{H}$, Bird $G$, Fisher $P$, et al. Coeliac disease in primary care: case finding study. BMJ 1999;318:164-7.

13. Rubin GP, Hungin AP, Kelly PJ, et al. Inflammatory bowel disease: epidemiology and management in an English general practice population. Aliment Pharmacol Ther 2000;14:1553-9.

14. Rubin GP, Hungin APS, Chinn DJ, et al. Quality of life in patients with established inflammatory bowel disease: a UK general practice survey. Aliment Pharmacol Ther 2004;19:529-35.

15. Fry J. Common diseases. Lancaster: MTP Press, 1979.

16. Jones RH, Lydeard S. Dyspepsia in the community: a follow-up study. Br J Clin Pract 1992; 46:95-7.

17. Agreus L, Svardsudd K, Nyren 0, et al. Irritable bowel syndrome and dyspepsia in the general population: overlap and lack of stability over time. Gastroenterology 1995; 109:671-80.

18. Muris JW, Starmans R, Fijten GH, et al. One-year prognosis of abdominal complaints in general practice: a prospective study of patients in whom no organic cause is found. Br J Gen Pract 1996;46:715-9.

19. Wallander MA, Johansson S, Ruigomez A, et al. Dyspepsia in general practice: incidence, risk factors, comorbidity and mortality. Fam Pract 2007b;24:403-11.

20. Jones R, Latinovic R, Charlton J, et al. Physical and psychological co-morbidity in irritable bowel syndrome: a matched cohort study using the General Practice Research Database. Aliment Pharmacol Ther 2006;24:879-6.

21. Ruigomez A, Garcia Rodriguez LA, Wallander MA, et al. Gastroesophageal reflux disease and asthma: a longitudinal study in UK general practice. Chest 2005;128:85-93

22. Lydeard S, Jones RH. Factors affecting the decision to consult with dyspepsia: comparison of consulters and non-consulters. J R Coll Gen Pract 1989;39:495-8.

23. Kettell J, Jones RH, Lydeard S. Reasons for consultation in irritable bowel syndrome: symptoms and patient characteristics. Br J Gen Pract 1992;42:459-61. 
24. Koloski NA, Boyce PM, Talley NJ. Is health care seeking for irritable bowel syndrome and functional dyspepsia a socially learned response to illness? Dig Dis Sci 2005;50:153-62.

25. Koloski NA, Boyce PM, Talley NJ. Somatization an independent psychosocial risk factor for irritable bowel syndrome but not dyspepsia: a population-based study. Eur J Gastroenterol Hepatol 2006;18:1101-9.

26. Hungin AP, Rubin GP, O'Flanagan $\mathrm{H}$. Long-term prescribing of proton pump inhibitors in general practice. Br J Gen Pract 1999;49:451-3.

27. Raghunath AS, Hungin APS, Cornford CS, et al. Use of proton pump inhibitors: an exploration of the attitudes, knowledge and perceptions of general practitioners. Digestion 2005; 72:212-8.

28. Armstrong D, Reyburn H, Jones R. A study of general practitioners' reasons for changing their prescribing behaviour. BMJ 1996;312:949-52.

29. Shaw MJ, Talley NJ, Beebe TJ, et al. Initial validation of a diagnostic questionnaire for gastroesophageal reflux disease. Am J Gastroenterol 2001;96:52-7.

30. Jones R, Coyne K, Wiklund I. The gastro-oesophageal reflux disease impact scale - a patient management tool for primary care. Aliment Pharmacol Ther 2007;25:1451-9.

31. Longstreth GF, Thompson WG, Chey WD, et al. Functional bowel disorders. Gastroenterology 2006;130:1480-92.

32. Jones RH. Upper gastrointestinal endoscopy - a view from general practice. J R Coll Gen Pract 1986;36:6-8.

33. Bytzer $\mathbf{P}$, Hansen JM, Schaffalitzky de Muckadell OB. Empirical H2-blocker therapy or prompt endoscopy in management of dyspepsia. Lancet 1994;343:811-6.

34. Lassen AT, Pedersen FM, Bytzer P, et al. Helicobacter pylori test-and-eradicate versus prompt endoscopy for management. Lancet 2000;356:455-60.

35. Jones R, Latinovic R, Charlton J, et al. Alarm symptoms in the early diagnosis of cancer in primary care. BMJ 2007;334:1040.
36. Johansson T, Petersen $\mathrm{H}$, Kristensen $\mathrm{P}$, et al. Cimetidine on-demand in dyspepsia. Experience with randomized controlled single-subject trials. Scan J Gastroenterol 1992;27:189-95.

37. Jones $\mathbf{R H}$, Baxter $\mathrm{G}$. Lansoprazole $30 \mathrm{mg}$ daily versus ranitidine $150 \mathrm{mg}$ bd in the treatment of acid-related dyspepsia in general practice. Aliment Pharmacol Ther 1997;11:541-6.

38. Jones $\mathbf{R}$, Crouch SL. Low-dose lansoprazole provides greater relief of heartburn and epigastric pain than low-dose omeprazole in patients with acid-related dyspepsia. Aliment Pharmacol Ther 1999;13:413-9.

39. Hungin AP, Rubin G, O'Flanagan $\mathrm{H}$. Factors influencing compliance in long-term proton pump inhibitor therapy in general practice. $\mathrm{Br} J$ Gen Pract 1999;49:463-4.

40. Jones RH, Holtmann G, Rodrigo L, et al. Alosetron relieves pain and improves bowel function compared with mebeverine in female nonconstipated irritable bowel syndrome patients. Aliment Pharmacol Ther 1999;13:1419-27.

41. Kennedy T, Jones R, Darnley S, et al. Cognitive behaviour therapy in addition to antispasmodic treatment for irritable bowel syndrome in primary care: randomized controlled trial. BMJ 2005;331:435-7.

42. McCrone P, Knapp M, Kennedy T, et al. Cost-effectiveness of cognitive behaviour therapy in addition to mebeverine for irritable bowel syndrome. Eur J Gastroenterol Hepatol 2008;20:255-63.

43. British Society of Gastroenterology. Guidelines on the irritable bowel syndrome. mechanisms and practical management. http://gut.bmj.com/onlinefirst.dtl. May 2007.

44. Kennedy TM, Jones RH. The epidemiology of hysterectomy and irritable bowel syndrome in a UK population. Int J Clin Pract 2000;54:647-60.

45. Kennedy TM, Jones RH. Epidemiology of cholecystectomy and irritable bowe syndrome in a UK population. Br J Surgery 2000;12:1658-64.

\section{Take advantage of BMJ Journals' remarkable catalogue of titles with Related Collections}

No busy professional has time to browse through all pertinent journals to find relevant articles, but with Related Collections you no longer have to. Follow the "Related Collections" link from any article and use the "Show Collections from other Journals" to expand your search across all BMJ Journals. Or simply follow the "Browse by topic" link on the home page. By setting up your own collections and receiving email alerts every time an article is added to your chosen area, you can build up your own significant body of knowledge. 